\title{
Untersuchungen zur Körperzusammensetzung und zum Wachstum von Kälbern unterschiedlicher genetischer Herkunft mittels Dualenergie-Röntgenabsorptiometrie (DXA)
}

\author{
Herrn Professor Dr. Peter Horst zum 75. Geburtstag gewidmet
}

\begin{abstract}
Title of the paper: In vivo analysis of body composition and growth of calves of different genetic origin using dualenergy $x$-ray absorptiometry (DXA)

In vivo body composition and growth of 214 calves (6-50 days old) of different genetic origin were analyzed using dual-energy x-ray absorptiometry (DXA). A whole body scan was performed. Looking at body composition there were differences among calf genotypes and parent genotypes. Purebred German-Holstein calves showed lower bone mineral density, bone mineral content, bone mineral percentage, fat tissue percentage and weight than the other genotypes. Opposite conditions existed for lean tissue percentage. Daily feed intake and tissue growth rates (lean tissue, fat tissue, bone mineral) were recorded from day 0 to day 50 of age. Further effects of birth type and lactation number of dam became obvious. Twins showed a significant higher daily gain than calves of single births. Heifer calves had highest daily gain between Scan 2 and Scan 3 in contrary to calves of cows of fourth or fifth lactation number.

Within the study two scan modes were compared. A comparison between two scan modes 'normal' and 'pediatric large' showed a high correlation of selected values $(r \geq 0,90)$ except for lean tissue percentage $(r=$ $0,62)$, fat tissue percentage $(r=0,70)$ and $R$-value $(r=0,69)$.

In conclusion DXA is a very suitable method for examination of body composition and growth of calves.
\end{abstract}

Key Words: dual-energy x-ray absorptiometry, calves, body composition, growth

\section{Zusammenfassung}

Die vorliegende Arbeit untersucht die Körperzusammensetzung und das Wachstum von 214 Kälbern (6 - 50 Tage alt) unterschiedlicher genetischer Herkunft in vivo mittels Dualenergie-Röntgenabsorptiometrie (DXA). Bezüglich der Körperzusammensetzung sind Unterschiede zwischen den Genotypen der Kälber bzw. der Elterntiere festgestellt worden. So waren reinrassige Deutsche-Holstein Kälber hinsichtlich Knochenmineraldichte, Knochenmineralgehalt, Knochenmineralanteil, Fettgewebsanteil und Masse den anderen Genotypen unterlegen. Umgekehrte Verhältnisse lagen beim Magerweichgewebsanteil vor. Im Rahmen der Wachstumsuntersuchung wurde neben den Gewebezunahmen (Magerweichgewebe, Fettgewebe, Knochenmineral) auch der Futterverbrauch untersucht. Des Weiteren wurden hier Effekte des Geburtstyps und der Laktationsnummer der Muttertiere deutlich. So wiesen Zwillingskälber signifikant höhere tägliche Gewichtszunahmen auf als Kälber aus Einlingsgeburten. Kälber von Jungkühen (1. Laktation) zeigten die höchsten täglichen Zunahmen zwischen 2. und 3. Scan, wohingegen Kälber von Viert- bzw. Fünftlaktierenden am wenigsten zunahmen.

Eine Analyse verschiedener Scan Modi „normal“ und „Pediatrie groß“ ergab eine sehr gute Korrelation der Messwerte $(r \geq 0,90)$ mit Ausnahme von Magerweichgewebe- $(r=0,62)$, Fettgewebeanteil $(r=0,70)$ und R-Wert $(r=0,69)$. Zusammenfassend ist das DXA-Verfahren als eine geeignete Möglichkeit zur Ermittlung der Körperzusammensetzung und des Wachstums bei Kälbern zu beurteilen.

Schlüsselwörter: Dualenergie-Röntgenabsorptiometrie, Kälber, Körperzusammensetzung, Wachstum

1. Einleitung

Die Dualenergie-Röntgenabsorptiometrie (DXA) als Untersuchungsmethode in der Erforschung von Körperzusammensetzungen bei Tieren ist ein immer häufiger ver- 
wendetes Verfahren. Die ursprünglich für die Humanmedizin entwickelte Analysemethode wird dort vor allem im Bereich der Osteoporosediagnostik eingesetzt.

In der Veterinärmedizin liegen mittlerweile einige Forschungsarbeiten im Bereich der Kleintiere (SPEAKMAN et al., 2001) und kleiner Heimtiere (FINK et al., 2002; TAMADA, 1998; BERTIN et al., 1998; GALA PANIAGUA et al., 1998) vor. Bei den landwirtschaftlichen Nutztieren sind bisher vor allem Schafe (TURNER et al.; 1995; POUILLES et al., 2000), Geflügel (MITCHELL et al., 1997; SWENNEN et al.2004) und Schweine (MITCHELL and SCHOLZ, 1997; MITCHELL et al., 1998; PICAUD et al., 1999; HAMMAMI et al., 2001; MITCHELL et al., 2001; MITCHELL et al., 2002; SCHOLZ et al., 2002; KOO et al., 2004) untersucht worden.

Intention dieser Arbeit ist es, im Rahmen eines Kreuzungsversuches zwischen den Rassen Deutsches Fleckvieh und Deutsche Holsteins das DXA Verfahren erstmals bei Kälbern in vivo einzusetzen, um damit Erkenntnisse über deren Wachstum und Körperzusammensetzung zu erlangen. Diese Studie ist Teil eines Projektes, das sich außerdem mit der Untersuchung des Klauenwachstums bei neugeborenen Kälbern befasst (NÜSKE et al., 2002) sowie in einer noch andauernden Studie Referenzuntersuchungen zwischen DXA-Analyse und Totalzerlegung (MUSICK, persönliche Mitteilung) befasst.

Die DXA-Untersuchung zählt zu den häufig in der Humanmedizin angewendeten Techniken zur Ermittlung der Körperzusammensetzung und zeigt eine sehr große Präzision und Genauigkeit. Diese werden von ELLIS (2001) mit 2-3\% bzw. 5\% für die Fettmasse angegeben. KOO et al. (2000) errechneten in ihrer Studie eine sehr hohe Korrelation $(\mathrm{r}=0,99)$ bei Ergebnissen von wiederholten DXA-Messungen. Dies spricht für eine sehr gute Wiederholbarkeit des Messverfahrens, die in Abhängigkeit von Gerät und Softwaremodus >75\% liegt (MITCHELL et al., 1998; SCHOLZ, 2002).

\subsection{Material und Methoden \\ $2.1 \quad$ Tiermaterial}

Insgesamt wurden 214 Kälber im Zeitraum von November 2000 bis August 2004 einer Untersuchung durch den DXA-Scanner (GE Lunar DPX IQ, Madison, WI) unterzogen. Der erste Scan fand in der ersten Lebenswoche der Kälber statt, gefolgt von zwei weiteren Untersuchungen jeweils im Abstand von drei Wochen. Scan 2 wurde mit 168 und Scan 3 mit 143 Kälbern durchgeführt. Verringerte Tierzahlen begründen sich vor allem durch Krankheit der Tiere zu den entsprechenden Terminen oder durch Verenden der Kälber.

Tabelle 1

Tiermaterial der verschiedenen Genotypen unterteilt in männlich und weiblich (Number of calves within the study of different genetic origin divided in male and female)

\begin{tabular}{lllllll}
\hline $\begin{array}{l}\text { Geschlecht } \\
\text { der Kälber }\end{array}$ & FVxFV & FVxKR & FVxDH & DHxFV & DHxKR & DHxDH \\
\hline männlich & 24 & 9 & 24 & 21 & 8 & 17 \\
weiblich & 20 & 12 & 16 & 25 & 19 & 19 \\
\hline gesamt & 44 & 21 & 40 & 46 & 27 & 36 \\
\hline
\end{tabular}

FV = Fleckvieh, DH = Deutsche Holsteins, KR = Kreuzung; (FV = German Fleckvieh, DH = German Holstein, KR = crossbred animal)

An der Studie waren Kälber der Rassen Deutsches Fleckvieh (FV), Deutsche Holstein (DH), F1-Kreuzungstiere (DHxFV bzw. FVxDH) und Rückkreuzungen (DHxKR, 
FVxKR) beteiligt. Eine Verteilung der Tierzahl nach Genotyp und Geschlecht findet sich in Tabelle 1.

Die Kälber stammten aus 148 verschiedenen Muttertieren, davon 52 Jungkühe und 96 Kühe (Laktationsnummer 2-5) sowie von 22 Vatertieren ( 9 DH, 13 FV). Auch die Muttertiere wurden entsprechend ihrer Rassezugehörigkeit in drei Gruppen unterteilt (Tab. 2).

Tabelle 2

Anzahl bei den Genotypen der Muttertiere; Kreuzungstiere = 25\%-75\% Deutsche Holsteins-Anteil (Number of mother genotypes; crossbreds = 25\%-75\% German Holstein percentage)

\begin{tabular}{lllll}
\hline Anzahl & Fleckvieh & Kreuzungstiere & Deutsche & Holsteins \\
\hline $\mathrm{n}$ & 58 & 41 & 49 & \\
\hline
\end{tabular}

Alle Kälber wurden im Lehr- und Versuchsgut Oberschleißheim geboren. Insgesamt stammten 34 Kälber aus Zwillingsgeburten und einer Drillingsgeburt, davon waren 22 weiblich und 12 männlich.

\subsection{Das DXA-Verfahren}

Die Grundlage der Dualenergie-Röntgenabsorptiometrie ist das Drei-KomponentenModell: Körpergewicht $=$ Magerweichgewebe + Fettgewebe + Knochenmineralstoffe . Daraus ergibt sich, dass Magerweichgewebe alles umfasst (Knorpel, innere Organe, Bindegewebe, etc.), was nicht Fettgewebe und Knochenmineralstoffe ist. PIETROBELLI et al. (1996) geben eine schematische Darstellung des Körper-Modells für DXA (Abb. 1).

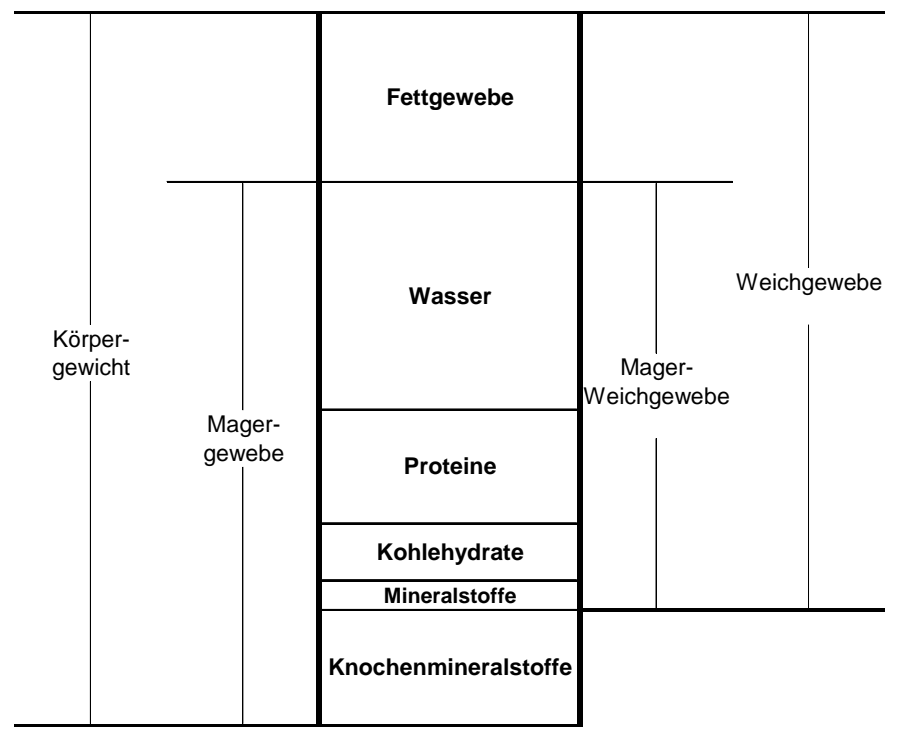

Abb. 1: Modell der Körperzusammensetzung nach PIETROBELLI (1996) (Model of body composition, modified after PIETROBELLI, 1996)

Die Funktionsweise des Dualenergie-Röntgenabsorptiometrie-Scanners basiert auf der Verwendung eines Ceriumphotonen-Filters, mit dem es möglich ist, die polyenergetische Röntgenstrahlung in zwei Energieniveaus, $38 \mathrm{keV}$ (Kiloelektronenvolt) und 70 keV, zu teilen. Die Messwerte entstehen durch die unterschiedliche Abschwächung der niedrig- und hochenergetischen Röntgenstrahlen durch Fett- oder andere Weichgewebe bzw. durch Knochen. Der Fett- und Magerweichgewebsgehalt eines jeden Mess- 
punktes, der kein Knochengewebe enthält, beruht auf dem Röntgenschwächungskoeffizienten für Weichgewebe (R-Wert), der unabhängig von der Gewebsdicke ist. Der theoretische R-Wert für Knochen beträgt 2,8617, für Lipide 1,2058 - 1,2333 und für Wasser 1,3572 (PIETROBELLI et al. 1996). Die Unterteilung in Knochen und Weichgewebe erfolgt über software-interne Algorithmen (SCHOLZ, 2002).

\subsection{Untersuchungsablauf}

Die Tiere wurden mittels einer Ketamin/Xylazin2\% Mischung intramusculär sediert (Dosierung 11,5 mg/kg bzw. 0,2 mg/kg). Danach folgte eine Gewichtsermittlung der Probanden auf der Balkenwaage. Für den DXA-Scan wurden die sedierten Tiere auf dem Scanner-Tisch in Brustlage gelagert.

Bei diesem Versuch handelt es sich um eine Ganzkörperanalyse im Scan Modus „normal“. 20 Kälber wurden zusätzlich im Scan Modus „Pediatrie groß“ gescannt. Die Unterschiede der beiden Scan Modi sind die Größe der Messpunkte, bei Modus „normal“ 4,8 x 9,6 mm und bei „Pediatrie groß“ 3,6 x 7,2 mm, und das Intervall der Meßpunkte, „normal“: 1/16 und „Pediatrie groß“: 1/32. Damit werden mehr und kleinere Punkte bei „Pediatrie groß“ gemessen, was zu einer abweichenden Berechnung der Parameter bei beiden Scan Modi führt.

Gemessen wurden Knochenmineraldichte (BMD in $\mathrm{g} / \mathrm{cm}^{2}$ ), Knochenmineralgehalt (BMC in g), Knochenmineralanteil (BMPC in \%), Weichgewebe (Soft in g) - bestehend aus Magerweichgewebe (Mager in g) und Fettgewebe (Fett in g) - Magerweichgewebsanteil (MagerPC in \%), Fettgewebsanteil (FettPC in \%) und das DXA Gewicht (DXWT in kg ).

\section{$2.4 \quad$ Haltung und Fütterung}

Die Kälber wurden direkt nach der Geburt in Kälberiglus außerhalb des Stalles verbracht, wo sie die ersten drei Tage dreimal täglich zwei Liter individuelles Kolostrum erhielten. Ab dem 4. Lebenstag bekamen die Tiere zweimal täglich drei Liter Vollmilch. Nach zwei Wochen erfolgte eine Umstallung der Kälber in einen Kaltstall mit Tiefstreu und Auslauf in Gruppenhaltung. Dort wurde Tränke (Milchaustauscher Bullilac 50 der Fa. Inntaler Milchfutter GmbH \& Co. KG, Zusammensetzung: 22,5\% Rohprotein, 19\% Rohfett und 50\% Magermilchpulver) und Kraftfutter automatisch sowie in Abhängigkeit vom Alter der Tiere zugeteilt (Tränke- und Kraftfutterautomat: Fa. Westphalia). Das pelletierte Kraftfutter bestand aus 28,67\% Weizen, 42,33\% Нafer, 28\% Soja und 1\% Mineralfutter. Zudem erhielten die Kälber Grundfutter, bestehend aus Mais- und Grassilage sowie Heu aus dem Futterbarren. Wasser stand ad libitum zur Verfügung.

\section{$2.5 \quad$ Statistische Auswertung}

Es wurde eine Varianzanalyse mit Hilfe des Statistikpaketes SAS 8.2 unter Verwendung des generalisierten linearen Modells (GLM) durchgeführt.

Folgende Modelle wurden zur Berechnung verwendet:

Für die Merkmale der Körperzusammensetzung wurde folgendes Modell angewendet:

$$
\mathrm{Y}_{\mathrm{ijklmnop}}=\underset{\substack{\mathrm{e} \\ \mathrm{e}_{\mathrm{ijklmnop}}}}{\mu} \mathrm{GV}_{\mathrm{i}}+\mathrm{GM}_{\mathrm{j}}+\left(\mathrm{GV}_{\mathrm{i}} \mathrm{x} \mathrm{GM}_{\mathrm{i}}\right)+\mathrm{G}_{\mathrm{k}}+\mathrm{M}_{\mathrm{l}}+\mathrm{L}_{\mathrm{m}}+\mathrm{MJ}_{\mathrm{n}}+\mathrm{MN}_{\mathrm{o}}+
$$




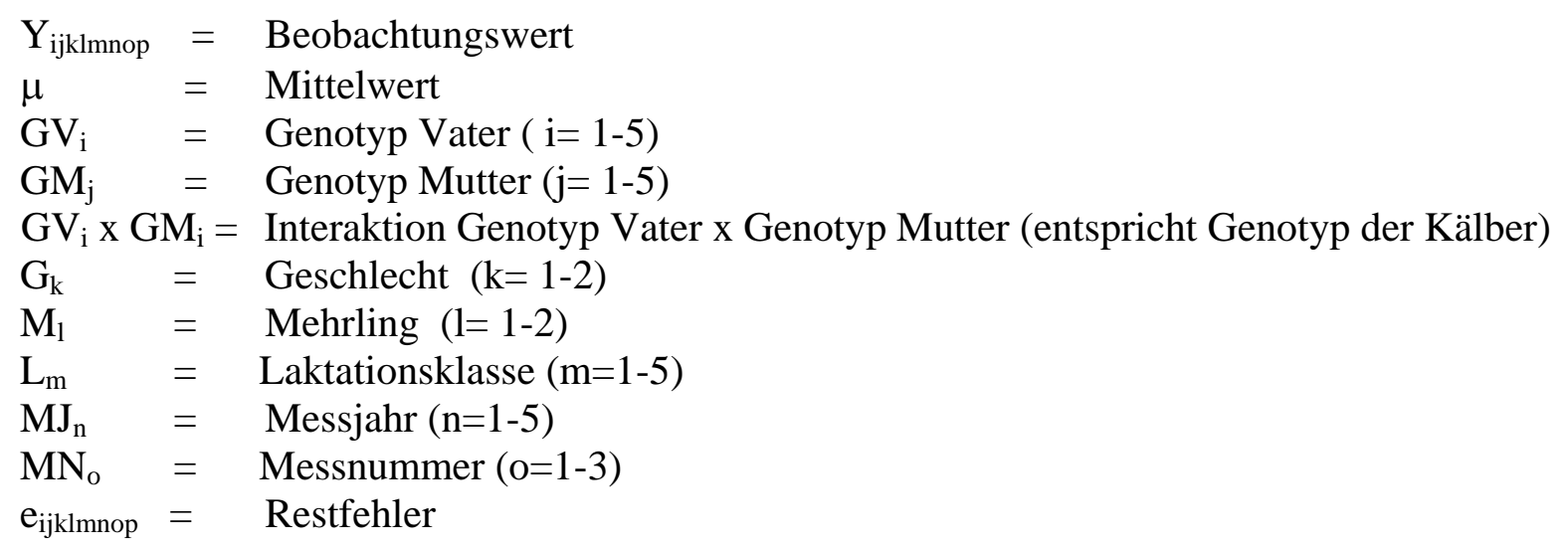

Für die Analyse der Zunahmen und des Futterverbrauches kam folgendes Modell zur Anwendung :

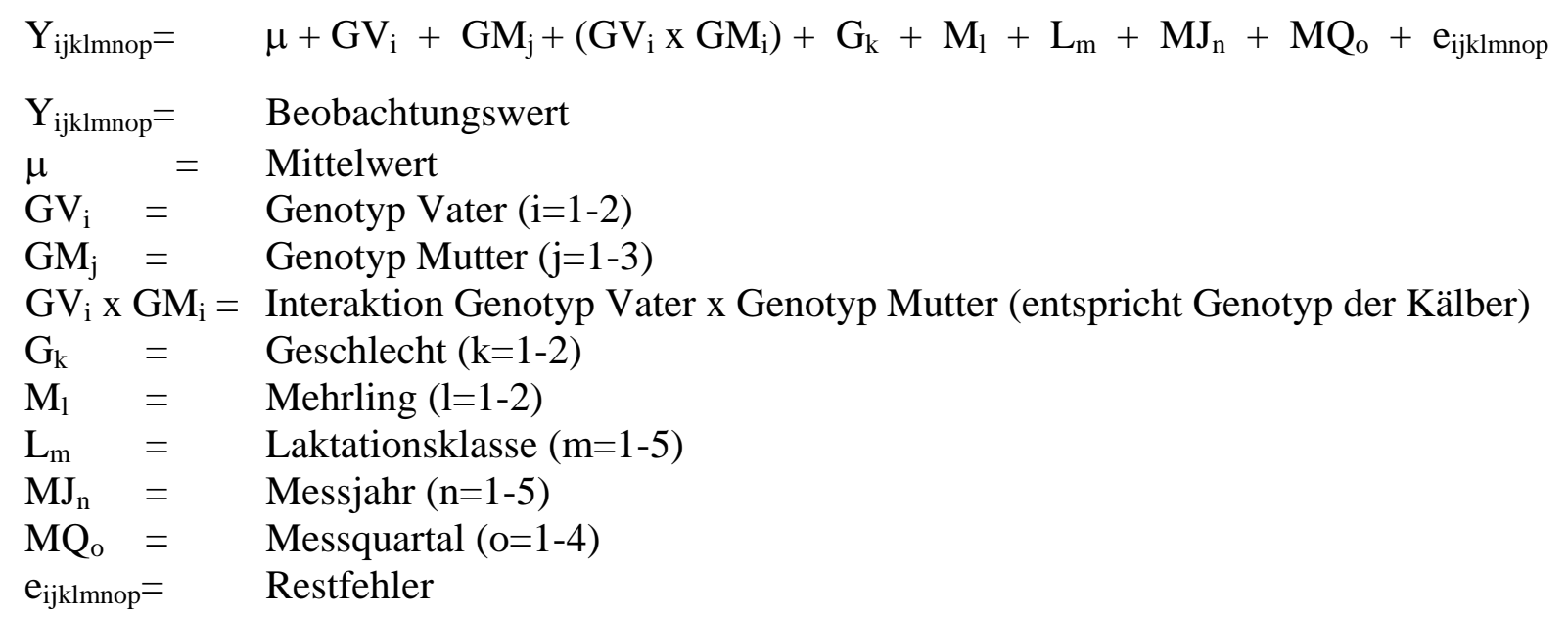

Für den Vergleich der beiden Scan-Modi wurde eine lineare Regression berechnet, in der die Werte für das Bestimmtheitsmaß $\left(\mathrm{R}^{2}\right)$ und der „Standard Error of Estimation“ (SEE) als Anhaltspunkte dienen.

Außerdem fand eine Korrektur der Werte statt, die den Weichgewebeanteil (Magerweichgewebe bzw. Fettgewebe) betreffen. Nötig war diese Korrektur, da für den DXA - Fettgehalt (\%) eine software-interne Schwellenwertfestsetzung existiert, die bei $\geq$ 3,8\%, bezogen auf das DXA-Gewicht, liegt. Die Abbildung 2 zeigt, dass bei vielen Messungen ( $n=235)$ - von insgesamt 519 Messungen - die gemessenen R-Werte die Schwelle von 1,39 überschritten. Dadurch wurde der ermittelte Fettgehalt softwareintern höher angegeben als entsprechend einer linear fortgesetzten Berechnung zu erwarten gewesen wäre. Deshalb wurden o.g. Werte anhand der R-Werte mit folgender Regressionsgleichung korrigiert: DXA - Fett (\%) korr. = 660,4311 - (472,3975 x RWert)

Diese Gleichung entstammt aus DXA-Untersuchungen beim Schwein. Hier liegen zahlreiche Messungen ( $\mathrm{n}>200$ ) vor, aus denen hervorgeht, dass eine sehr hohe Korrelation zwischen FettPC und R-Wert besteht $(r>0,99)$, wenn der Fettanteil über 3,8\% liegt (SCHOLZ, 2002). 


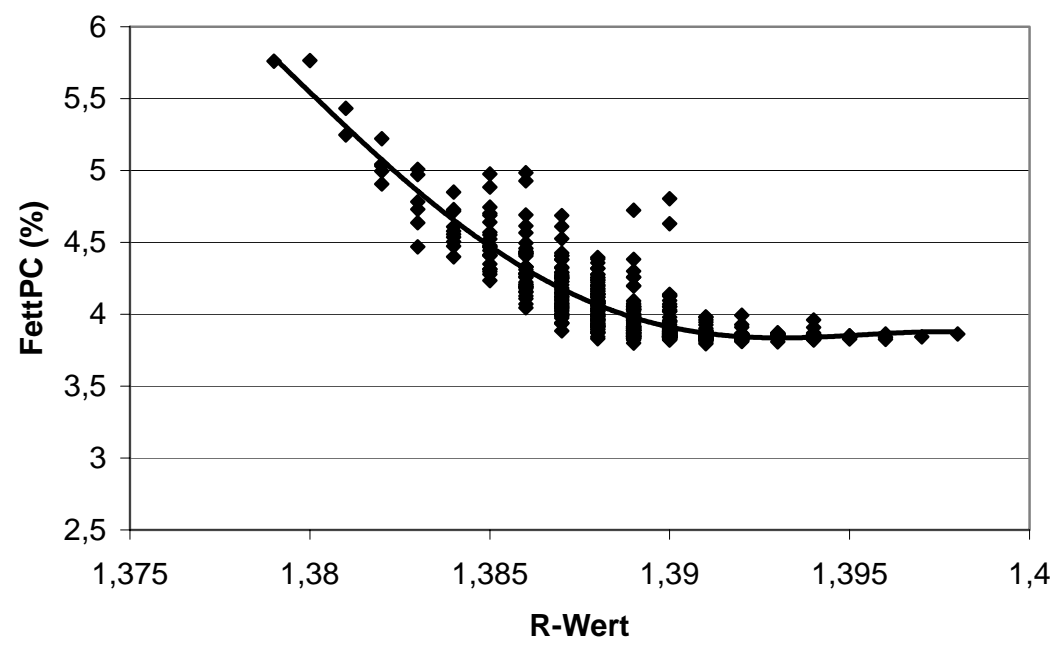

Abb. 2: Beziehung zwischen R-Wert und Fettgewebsanteil vor Korrektur der Werte (Relation of R-value and fat percentage without correction)

Die Regressionsgleichung aus den Schweinedaten wurde so modifiziert, dass im beobachteten Parameterraum von R keine negativen Fettgehaltswerte beim Kalb auftreten. Abbildung 3 zeigt den Verlauf nach der Korrektur.

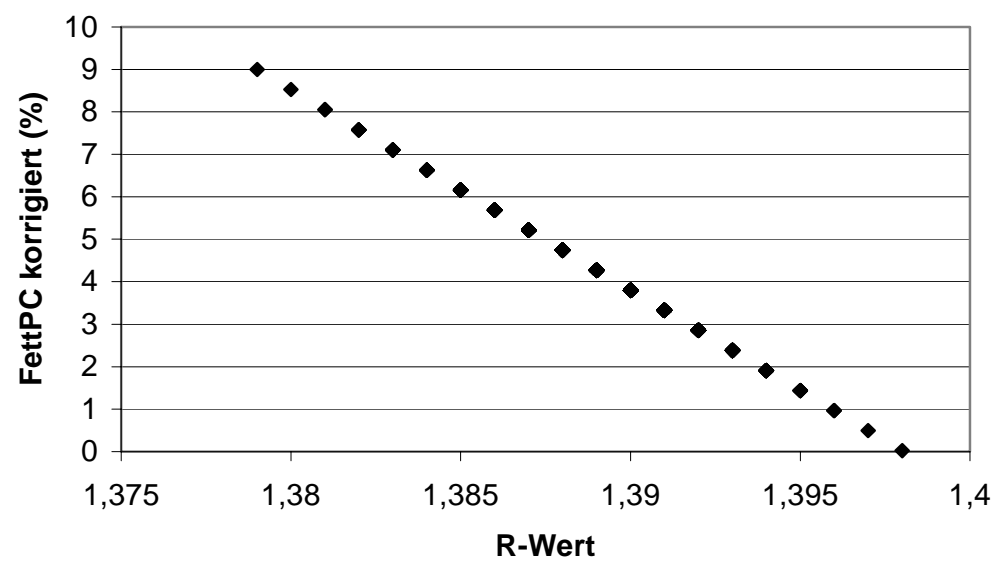

Abb. 3: Beziehung zwischen R-Wert und Fettgewebsanteil nach Korrektur der Werte (Relation of R-value and fat percentage after correction)

3. $\quad$ Ergebnisse

3.1 Effekt des Genotyps der Kälber (s. Tab. 3)

Diese Ergebnisse basieren auf der Auswertung aller drei DXA-Untersuchungen und geben somit einen Durchschnitt der einzelnen Parameter zwischen 6. und 50. Lebenstag der Kälber wieder.

Bei Betrachtung der Ergebnisse von BMD, BMC, BMPC und DXWT wird deutlich, dass reinrassige $\mathrm{DH}$-Kälber signifikant die niedrigsten Werte erreichen. Beim Magerweichgewebsanteil erzielen sie signifikant höhere Prozentanteile als reinrassige Fleckvieh- und DHxFV-Kälber. Außerdem sinkt mit zunehmendem DH-Anteil sowohl das Körpergewicht als auch der Fettanteil. Reine Fleckviehkälber verfügen in der Tendenz über den höchsten Körperfettgehalt (\%). 
Tabelle 3

Effekt des Genotyps der Kälber (kleinste Quadrate Mittelwerte \pm Standardschätzfehler) (Effect of calf genotype (Least Squares Means \pm Standard Error of Estimation))

\begin{tabular}{lllllll}
\hline Genotyp & $\begin{array}{l}\text { Knochen- } \\
\text { mineraldichte } \\
\left(\mathrm{g} / \mathrm{cm}^{2}\right)\end{array}$ & $\begin{array}{l}\text { Knochen- } \\
\text { mineralgehalt } \\
(\mathrm{g})\end{array}$ & $\begin{array}{l}\text { Knochen- } \\
\text { mineralanteil } \\
(\%)\end{array}$ & $\begin{array}{l}\text { Magerweich- } \\
\text { gewebsanteil } \\
(\%)\end{array}$ & $\begin{array}{l}\text { Fettge- } \\
\text { websanteil (\%) }\end{array}$ & $\begin{array}{l}\text { DXA-Gewicht } \\
(\mathrm{kg})\end{array}$ \\
\hline FVxFV & $0,978 \pm 0,008^{\mathrm{bc}}$ & $2025,6 \pm 30,8^{\mathrm{b}}$ & $3,82 \pm 0,04^{\mathrm{bc}}$ & $91,65 \pm 0,13^{\mathrm{b}}$ & $4,53 \pm 0,12^{\mathrm{b}}$ & $53,23 \pm 0,62^{\mathrm{bcd}}$ \\
FVxKR & $0,981 \pm 0,012^{\mathrm{bc}}$ & $2070,2 \pm 45,4^{\mathrm{b}}$ & $3,84 \pm 0,06^{\mathrm{bc}}$ & $91,81 \pm 0,19^{\mathrm{ab}}$ & $4,35 \pm 0,18^{\mathrm{ab}}$ & $54,04 \pm 0,92^{\mathrm{bcd}}$ \\
FVxDH & $0,964 \pm 0,009^{\mathrm{b}}$ & $2034,8 \pm 34,6^{\mathrm{b}}$ & $3,74 \pm 0,04^{\mathrm{ab}}$ & $91,83 \pm 0,15^{\mathrm{ab}}$ & $4,43 \pm 0,14^{\mathrm{ab}}$ & $54,61 \pm 0,70^{\mathrm{c}}$ \\
DHxFV & $0,992 \pm 0,008^{\mathrm{c}}$ & $2047,8 \pm 29,7^{\mathrm{b}}$ & $3,90 \pm 0,04^{\mathrm{c}}$ & $91,75 \pm 0,13^{\mathrm{b}}$ & $4,34 \pm 0,12^{\mathrm{ab}}$ & $52,69 \pm 0,60^{\mathrm{bd}}$ \\
DHxKR & $0,958 \pm 0,011^{\mathrm{b}}$ & $1989,8 \pm 39,9^{\mathrm{b}}$ & $3,85 \pm 0,05^{\mathrm{bc}}$ & $91,88 \pm 0,17^{\mathrm{ab}}$ & $4,27 \pm 0,16^{\mathrm{ab}}$ & $52,06 \pm 0,80^{\mathrm{ab}}$ \\
DHxDH & $0,921 \pm 0,009^{\mathrm{a}}$ & $1829,9 \pm 33,7^{\mathrm{a}}$ & $3,65 \pm 0,04^{\mathrm{a}}$ & $92,18 \pm 0,14^{\mathrm{a}}$ & $4,17 \pm 0,14^{\mathrm{a}}$ & $50,48 \pm 0,68^{\mathrm{a}}$ \\
\hline
\end{tabular}

Unterschiedliche Buchstaben bedeuten signifikante Unterschiede ( $\mathrm{p} \leq 0,05)$.

Different superscripts show significant differences $(\mathrm{p} \leq 0,05)$. 4, 5 und 6). Kälber von FV-Vätern erlangen bei allen Parametern höhere Werte als von DH-Vätern.

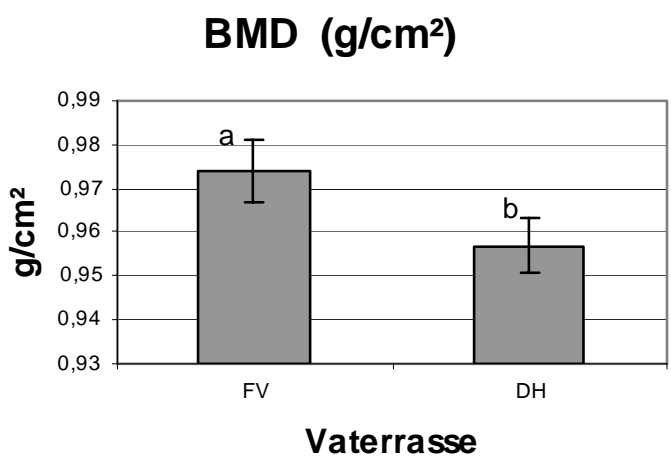

Abb. 4: Effekt der Vaterrasse auf die Knochenmineraldichte; $\mathrm{FV}=$ Fleckvieh, $\mathrm{DH}=$ Deutsche Holsteins; Unterschiedliche Buchstaben bedeuten signifikante Unterschiede $(\mathrm{p} \leq 0,05)$ (Effect of sire genotype on bone mineral density; FV = German Fleckvieh, DH =
German Holstein; Different superscripts show significant differences $(\mathrm{p} \leq 0,05))$
BMC (g)

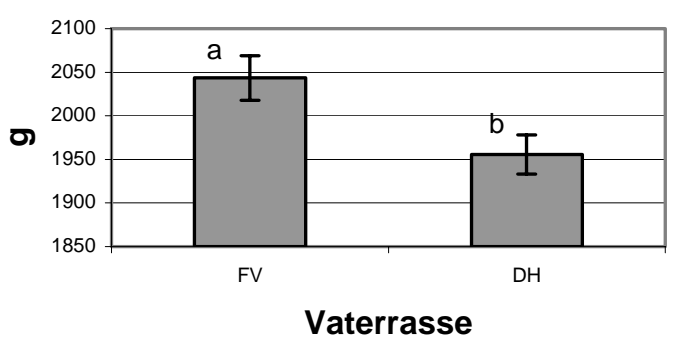

Abb. 5: Effekt der Vaterrasse auf den Knochenmineralgehalt; FV = Fleckvieh, $\mathrm{DH}=$ Deutsche Holsteins; Unterschiedliche Buchstaben bedeuten signifikante Unterschiede $(p \leq 0,05)$ (Effect of sire genotype on bone mineral content; $\mathrm{FV}=$ German Fleckvieh, $\mathrm{DH}=$ German Holstein; Different superscripts show significant differences $(\mathrm{p} \leq 0,05))$ 
DXWT (kg)

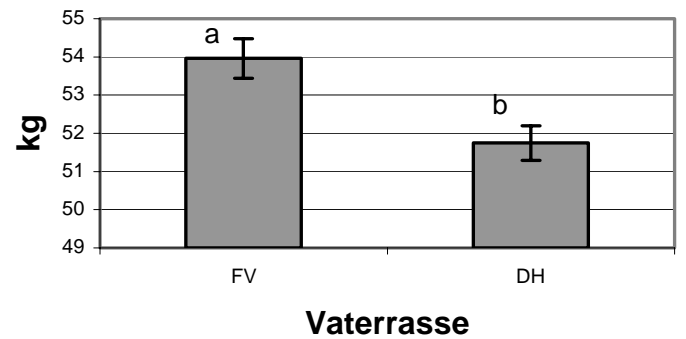

Abb. 6: Effekt der Vaterrasse auf das DXA-Gewicht; $\mathrm{FV}=$ Fleckvieh, $\mathrm{DH}=$ Deutsche Holsteins; Unterschiedliche Buchstaben bedeuten signifikante Unterschiede $(\mathrm{p} \leq 0,05)$ (Effect of sire genotype on DXAweight; $\mathrm{FV}=$ German Fleckvieh, $\mathrm{DH}=$ German Holstein; Different superscripts show significant differences $(\mathrm{p} \leq 0,05))$

\subsubsection{Effekt der Mutterrasse}

Auch der Muttergenotyp hat einen signifikanten Einfluss auf BMD und BMC sowie auf BMPC (Abb. 7 - 9), wobei Kälber von DH-Müttern niedrigere Werte erreichen als von FV- bzw. Kreuzungsmüttern. Eine Tendenz im Einfluss der Mutterrasse (Abb. 10) ist bei MagerPC $(p=0,0574)$ zu erkennen. Hier erzielen Kälber von DH-Müttern signifikant größere Prozentanteile als von FV-Müttern.

\section{BMD $\left(\mathbf{g} / \mathrm{cm}^{2}\right)$}

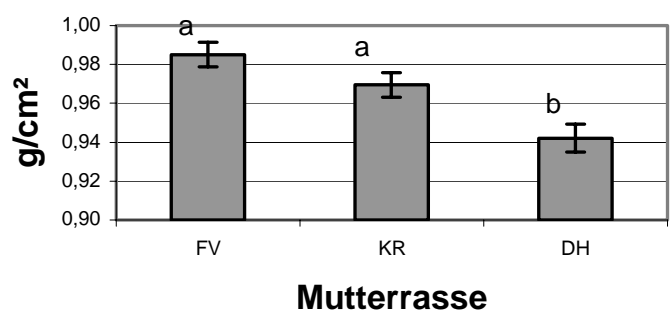

$\operatorname{BMC}(g)$

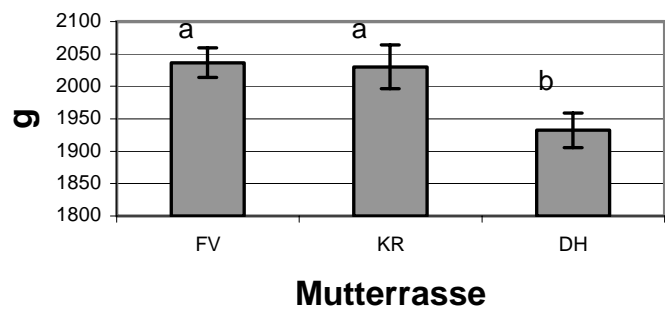

Abb. 7: Effekt der Mutterrasse auf die Knochenmine- Abb. 8: Effekt der Mutterrasse auf den Knochenmineraldichte; FV = Fleckvieh, $\mathrm{KR}=$ Kreuzungstiere, $\mathrm{DH}=$ ralgehalt; FV = Fleckvieh, $\mathrm{KR}=$ Kreuzungstiere, $\mathrm{DH}=$ Deutsche Holsteins; Unterschiedliche Buchstaben be- Deutsche Holsteins; Unterschiedliche Buchstaben bedeuten signifikante Unterschiede $(\mathrm{p} \leq 0,05)$ (Effect of deuten signifikante Unterschiede $(\mathrm{p} \leq 0,05)$ (Effect of mother genotype on bone mineral density; $\mathrm{FV}=$ mother genotype on bone mineral content; $\mathrm{FV}=$ German Fleckvieh, $\mathrm{KR}=$ crossbred animals, $\mathrm{DH}=$ German Fleckvieh, $\mathrm{KR}=$ crossbred animals, $\mathrm{DH}=$ German Holstein; Different superscripts show German Holstein; Different superscripts show significant differences $(\mathrm{p} \leq 0,05))$ significant differences $(\mathrm{p} \leq 0,05))$ 
BMPC (\%)

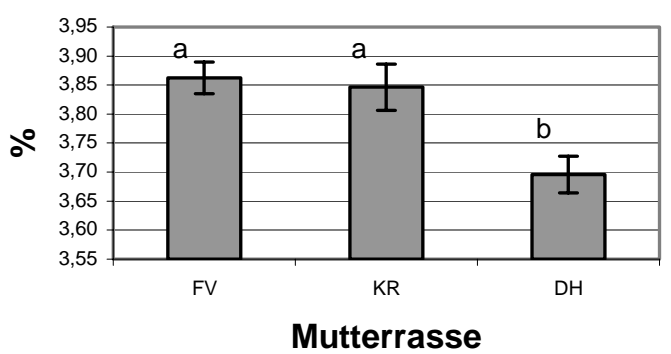

Mager PC (\%)

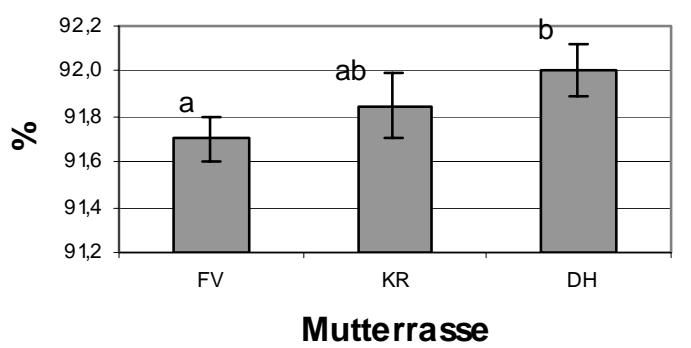

Abb. 9: Effekt der Mutterrasse auf den Knochenmine- Abb. 10: Effekt der Mutterrasse auf den Magerweichralanteil; FV = Fleckvieh, KR = Kreuzungstiere, $\mathrm{DH}=$ gewebsanteil; FV = Fleckvieh, KR = Kreuzungstiere, Deutsche Holsteins; Unterschiedliche Buchstaben be- DH = Deutsche Holsteins; Unterschiedliche Buchstadeuten signifikante Unterschiede $(p \leq 0,05)$ (Effect of ben bedeuten signifikante Unterschiede $(p \leq 0,05)$ mother genotype on bone mineral percentage; $\mathrm{FV}=$ (Effect of mother genotype on lean tissue percentage; German Fleckvieh, KR = crossbred animals, DH $=$ FV $=$ German Fleckvieh, KR = crossbred animals, DH German Holstein; Different superscripts show = German Holstein; Different superscripts show significant differences $(\mathrm{p} \leq 0,05))$

significant differences $(\mathrm{p} \leq 0,05))$

3.3. Wachstum der Kälber (unterteilt in Abschnitte 1.-2. Scan und 2.-3. Scan)

\subsection{1.} Futterverbrauch

Bei diesen Ergebnissen ist zu beachten, dass hier nur die Kraftfuttermenge pro Tag betrachtet wird, da die Kälber unterschiedlich früh mit der Aufnahme zusätzlich zum Milchaustauscher beginnen. Vollmilch und Milchaustauscher werden rationiert und für Kälber des gleichen Alters in gleicher Menge gefüttert, so dass dadurch keine signifikanten Unterschiede im Verbrauch existierten. Die Grundfutteraufnahme wird nur als durchschnittlicher Wert für alle Kälber angegeben (Mais- und Grassilage $=0,37$ $\mathrm{kg} / \mathrm{Tag}$ und Heu $=0,21 \mathrm{~kg} / \mathrm{Tag}$ ) Dieses Ergebnis basiert auf der Erfassung der Verbrauchsdaten in einem Zeitraum von 17 Monaten, in denen täglich das Grundfutter in den Futterbarren eingewogen und am nächsten Morgen die Reste getrennt zurückgewogen wurden. Das Ergebnis der Subtraktion der Rückwaage von der Einwaage wurde durch die sich in der Bucht befindende Anzahl der Kälber geteilt.

\section{Kraftfutterverbrauch/Tag (kg)}

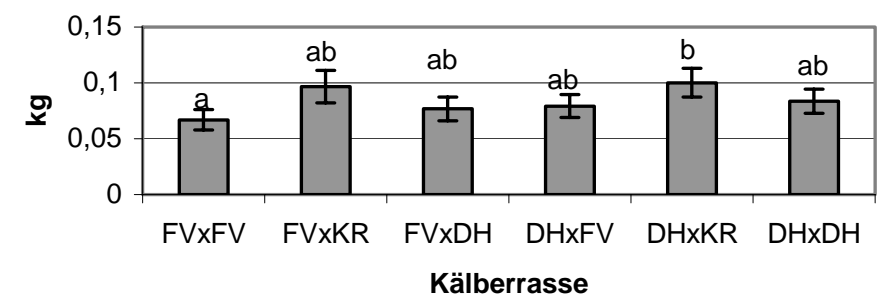

Abb. 11: Effekt des Genotyps der Kälber auf den täglichen Kraftfutterverbrauch zwischen 1. und 2. Scan; FV = Fleckvieh, DH = Deutsche Holsteins, KR = Kreuzungstiere; Unterschiedliche Buchstaben bedeuten signifikante Unterschiede $(\mathrm{p} \leq 0,05)$ (Effect of calf genotype on daily concentrate intake between scan 1 and 2; FV = German Fleckvieh, DH = German Holstein, KR = crossbred animals; Different superscripts show significant differences $(\mathrm{p} \leq 0,05))$

Bei der Kraftfutteraufnahme pro Tag zwischen den ersten beiden Scans (5. - 26. Lebenstag) zeigen sich signifikante Unterschiede des Verbrauchs zwischen den Genoty- 
pen der Kälber (Abb. 11), wobei reinrassige Fleckviehkälber signifikant weniger Kraftfutter aufnehmen als FVxKR- und DHxKR-Kälber.

Weiter beeinflusst die Rasse der Mutter in dieser Altersspanne tendenziell $(p=0,0703)$ den Kraftfutterverbrauch. Abbildung 12 zeigt, dass Kälber von Kreuzungsmüttern signifikant mehr fressen als Kälber von FV-Müttern. Die Vaterrasse hatte keinen signifikanten Einfluss auf die Kraftfutteraufnahme.

\section{Kraftfutterverbrauch/Tag (kg)}

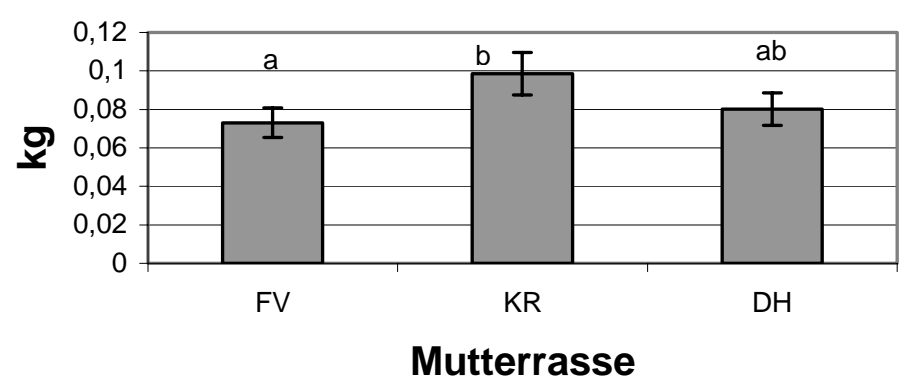

Abb. 12: Effekt der Mutterrasse auf den täglichen Kraftfutterverbrauch zwischen 1. und 2. Scan; FV = Fleckvieh, $\mathrm{KR}=$ Kreuzungstiere, $\mathrm{DH}$ = Deutsche Holsteins; Unterschiedliche Buchstaben bedeuten signifikante Unterschiede $(\mathrm{p} \leq 0,05)$ (Effect of mother genotype on daily concentrates intake between scan 1 and 2; FV = German Fleckvieh, KR = crossbred animals, DH = German Holstein; Different superscripts show significant differences $(\mathrm{p} \leq 0,05))$

Zwischen den letzten beiden Scans (27. - 48. Lebenstag) hat die Laktationsnummer einen signifikanten Einfluss auf die tägliche Kraftfutteraufnahme (Abb. 13). Kälber von Müttern der 3. Laktation nehmen signifikant weniger Kraftfutter auf als Kälber von Müttern der 1. oder 2. Laktation. Es existieren keine signifikanten Unterschiede in der Kraftfutteraufnahme/Tag zwischen den Kälbergenotypen bzw. den Genotypen der Eltern.

Kraftfutteraufnahme/Tag (kg)

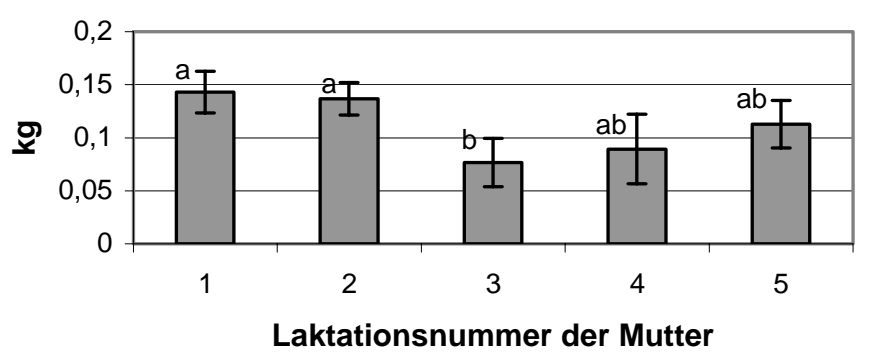

Abb. 13: Effekt der Laktationsnummer der Mutter auf den täglichen Kraftfutterverbrauch zwischen 2. und 3. Scan; Unterschiedliche Buchstaben bedeuten signifikante Unterschiede $(p \leq 0,05)$ (Effect of mothers lactation number on daily concentrates intake between scan 2 and 3; Different superscripts show significant differences $(\mathrm{p} \leq 0,05))$

\subsubsection{Gewebe-Zunahmen (unterteilt in Abschnitte 1.-2. Scan und 2.-3. Scan)}

Die täglichen Zunahmen zwischen Scan 1 und Scan 2 werden unterteilt in Fettgewebezunahme/Tag, Magerweichgewebezunahme/Tag, BMC-Zunahme/Tag und DXWTZunahme/Tag. Signifikanten Einfluss auf diese Parameter haben der Faktor Geburtstyp (s. Tab. 4) und die Laktationsnummer (s. Tab. 5). Dies gilt nicht für die Fettgewebezu- 
nahmen, bei denen das Geschlecht einen signifikanten Einfluss hat. Männliche Kälber nehmen tendenziell Fettgewebe ab (-10,45 \pm 5,67), während weibliche Kälber tendenziell Fettgewebe ansetzen (6,70 \pm 5,74).

Außerdem sind starke tendenzielle Unterschiede zwischen den Muttergenotypen bei den täglichen Fettgewebe-Zunahmen zu beobachten. Kälber von DH-Müttern nehmen innerhalb der ersten vier Lebenswochen Fettgewebe (-11,5 $\pm 6,3$ g) ab, während Kälber von FV- (2,2 $\pm 5,6 \mathrm{~g})$ und Kreuzungsmüttern (3,6 $\pm 8,3 \mathrm{~g})$ zunehmen. Die Vaterrasse hat keinen Einfluss auf die täglichen Gewebe-Zunahmen (Tab. 6).

\section{Tabelle 4}

Effekt des Geburtstyps auf die täglichen Gewebe-Zunahmen (in g) zwischen Scan 1 und Scan 2 (kleinste Quadrate Mittelwerte \pm Standardschätzfehler) (Effect of birth type on daily tissue gain (in g) between scan 1 and scan 2 (Least Squares Means \pm Standard Error of Estimation))

\begin{tabular}{lllll}
\hline Geburtstyp & $\begin{array}{l}\text { Fettgewebe-Zu- } \\
\text { nahme }\end{array}$ & $\begin{array}{l}\text { Magerweichgewebe- } \\
\text { Zunahme }\end{array}$ & $\begin{array}{l}\text { Knochenmineral- } \\
\text { Zunahme }\end{array}$ & $\begin{array}{l}\text { DXA-Gewicht- } \\
\text { Zunahme }\end{array}$ \\
\hline Einling & $-7,5 \pm 4,0$ & $301,9 \pm 14,3^{\text {a }}$ & $11,9 \pm 0,5^{\mathrm{a}}$ & $306,3 \pm 15,0^{\mathrm{a}}$ \\
Mehrling & $3,7 \pm 8,4$ & $404,1 \pm 29,7^{\mathrm{b}}$ & $13,9 \pm 1,0^{\mathrm{b}}$ & $421,8 \pm 31,2^{\mathrm{b}}$ \\
\hline Unterschiedliche Buchstaben bezeichnen signifikante Unterschiede $=\mathrm{p} \leq 0,05$ & (Different superscripts mark significant differences $=\mathrm{p} \leq 0,05)$
\end{tabular}

Tabelle 5

Effekt der Laktationsnummer auf die täglichen Gewebe-Zunahmen (in g) zwischen Scan 1 und 2 (kleinste Quadrate Mittelwerte \pm Standardschätzfehler) (Effect of lactation number on daily tissue gain (in g) between scan1 and scan 2 (Least Squares Means \pm Standard Error of Estimation))

\begin{tabular}{lllll}
\hline $\begin{array}{l}\text { Laktations- } \\
\text { nummer }\end{array}$ & $\begin{array}{l}\text { Fettgewebe- } \\
\text { Zunahme }\end{array}$ & $\begin{array}{l}\text { Magerweichgewebe- } \\
\text { Zunahme }\end{array}$ & $\begin{array}{l}\text { Knochenmineral- } \\
\text { Zunahme }\end{array}$ & $\begin{array}{l}\text { DXA-Gewicht- } \\
\text { Zunahme }\end{array}$ \\
\hline 1 & $-13,4 \pm 7,4^{\mathrm{a}}$ & $381,1 \pm 26,1^{\mathrm{b}}$ & $15,1 \pm 0,9^{\mathrm{a}}$ & $382,9 \pm 27,4^{\mathrm{ab}}$ \\
2 & $3,1 \pm 5,6^{\mathrm{ab}}$ & $347,7 \pm 20,0^{\mathrm{ab}}$ & $13,1 \pm 0,7^{\mathrm{b}}$ & $364,0 \pm 21,0^{\mathrm{abc}}$ \\
3 & $-4,0 \pm 7,6^{\mathrm{ab}}$ & $342,7 \pm 27,1^{\mathrm{ab}}$ & $12,8 \pm 0,9^{\mathrm{b}}$ & $351,5 \pm 28,4^{\mathrm{bc}}$ \\
4 & $-4,9 \pm 11,5^{\mathrm{ab}}$ & $282,8 \pm 40,8^{\mathrm{a}}$ & $8,6 \pm 1,4^{\mathrm{c}}$ & $286,5 \pm 42,8^{\mathrm{c}}$ \\
5 & $9,7 \pm 9,0^{\mathrm{b}}$ & $411,0 \pm 32,1^{\mathrm{b}}$ & $14,8 \pm 1,1^{\mathrm{ab}}$ & $435,4 \pm 33,7^{\mathrm{a}}$ \\
\hline
\end{tabular}

1 bis 5 = Laktationsnummer; unterschiedliche Buchstaben bezeichnen signifikante Unterschiede $=p \leq 0,05$ (1 to $5=$ lactation numbers; different superscripts mark significant differences $=\mathrm{p} \leq 0,05$ )

\section{Tabelle 6}

Effekt der Vaterrasse auf die täglichen Gewebe-Zunahmen (in g) zwischen Scan 1 und Scan 2 (kleinste Quadratmittelwerte \pm Standardschätzfehler) (Effect of sire genotype on daily tissue gain (in g) between scan 1 and scan 2 (Least Squares Means \pm Standart Error of Estimation))

\begin{tabular}{lllll}
\hline Vaterrasse & $\begin{array}{l}\text { Fettgewebe- } \\
\text { Zunahme }\end{array}$ & $\begin{array}{l}\text { Magerweichgewebe- } \\
\text { Zunahme }\end{array}$ & $\begin{array}{l}\text { Knochenmineral- } \\
\text { Zunahme }\end{array}$ & $\begin{array}{l}\text { DXA-Gewicht- } \\
\text { Zunahme }\end{array}$ \\
\hline DH-Vater & $-2,6 \pm 5,7$ & $370,3 \pm 20,1$ & $13,2 \pm 0,7$ & $380,7 \pm 21,1$ \\
FV-Vater & $-1,0 \pm 6,0$ & $335,8 \pm 21,3$ & $12,6 \pm 0,7$ & $347,4 \pm 22,4$ \\
\hline DH - Deursche
\end{tabular}

$\mathrm{DH}=$ Deutsche Holsteins, FV = Fleckvieh (DH = German Holstein, FV = German Fleckvieh)

\section{Tabelle 7}

Effekt der Laktationsnummer auf die täglichen Gewebe-Zunahmen (in g) zwischen Scan 2 und 3 (kleinste Quadrate Mittelwerte \pm Standardschätzfehler) (Effect of lactation number on daily tissue gain (in g) between scan 2 and 3 (Least Squares Means \pm Standard Error of Estimation))

\begin{tabular}{|c|c|c|c|c|}
\hline $\begin{array}{l}\text { Laktations- } \\
\text { nummer }\end{array}$ & $\begin{array}{l}\text { Fettgewebe- } \\
\text { Zunahme }\end{array}$ & $\begin{array}{l}\text { Magerweichgewebe- } \\
\text { Zunahme }\end{array}$ & $\begin{array}{l}\text { Knochenmineral- } \\
\text { Zunahme }\end{array}$ & $\begin{array}{l}\text { DXA-Gewicht- } \\
\text { Zunahme }\end{array}$ \\
\hline 1 & $42,4 \pm 9,5^{\mathrm{a}}$ & $425,0 \pm 35,9$ & $12,3 \pm 1,1$ & $479,8 \pm 38,3$ \\
\hline 2 & $12,0 \pm 7,3^{b}$ & $435,0 \pm 27,6$ & $11,9 \pm 0,9$ & $458,9 \pm 29,5$ \\
\hline 3 & $23,4 \pm 11,1^{\mathrm{ab}}$ & $422,6 \pm 42,1$ & $11,1 \pm 1,3$ & $457,1 \pm 45,0$ \\
\hline 4 & $18,3 \pm 16,0^{\mathrm{ab}}$ & $311,3 \pm 60,4$ & $10,4 \pm 1,9$ & $340,0 \pm 64,5$ \\
\hline 5 & $-0,4 \pm 11,0^{b}$ & $360,5 \pm 41,6$ & $9,7 \pm 1,3$ & $369,8 \pm 44,4$ \\
\hline
\end{tabular}

1 bis 5 = Laktationsnummer; unterschiedliche hochgestellte Buchstaben bezeichnen signifikante Unterschiede $=\mathrm{p} \leq 0,05(1-5=$ lactation numbers; different superscripts mark significant differences $=p \leq 0,05$ ) 
Zwischen Scan 2 und 3 herrscht ein signifikanter Einfluss der Laktationsklasse auf die Fettgewebe-Zunahmen/Tag (Tab. 7) und die Vaterrasse beeinflusst signifikant die Magerweichgewebe- und DXWT-Zunahmen (Tab. 8), wobei Kälber von FV-Vätern die höheren Zunahmen erreichen.

\section{Tabelle 8}

Effekt der Vaterrasse auf die täglichen Gewebezunahmen (in g) zwischen Scan 2 und Scan 3 (kleinste Quadrate Mittelwerte \pm Standardschätzfehler) (Effect of sire genotype on daily tissue gain (in g) between scan 2 and scan 3 (Least Squares Means \pm Standard Error of Estimation))

\begin{tabular}{lllll}
\hline Vaterrasse & $\begin{array}{l}\text { Fettgewebe- } \\
\text { Zunahme }\end{array}$ & $\begin{array}{l}\text { Magerweichgewebe- } \\
\text { Zunahme }\end{array}$ & $\begin{array}{l}\text { Knochenmineral- } \\
\text { Zunahme }\end{array}$ & $\begin{array}{l}\text { DXA-Gewicht- } \\
\text { Zunahme }\end{array}$ \\
\hline DH-Vater & $16,5 \pm 7,8$ & $353,7 \pm 29,4^{\mathrm{a}}$ & $10,6 \pm 0,9$ & $380,7 \pm 31,3^{\mathrm{a}}$ \\
FV-Vater & $21,8 \pm 8,2$ & $428,1 \pm 31,0^{\mathrm{b}}$ & $11,6 \pm 1,0$ & $461,5 \pm 33,1^{\mathrm{b}}$ \\
\hline
\end{tabular}

$\mathrm{DH}=$ Deutsche Holsteins, FV = Fleckvieh; Unterschiedliche hochgestellte Buchstaben bezeichnen signifikante Unterschiede = $\leq \leq 0,05$; $(\mathrm{DH}$ $=$ German Holstein, FV = German Fleckvieh; Different superscripts mark significant differences $=p \leq 0,05$ )

\subsection{Vergleich von Scan Modus „normal“ zu Scan Modus „Pediatrie groß“}

Es konnte eine sehr gute Korrelation zwischen den beiden Scan Modi für die Parameter BMD, BMC, BMPC, Magerweichgewebe, Fettgewebe und DXWT festgestellt werden (Tab. 9). Eine niedrigere Korrelation liegt für die prozentualen Werte von Magerweich- und Fettgewebe sowie den R-Werten vor. Offensichtlich beeinflusst der Scan-Modus (Pixelgröße und Scangeschwindigkeit) die gemessene Weichgewebeschwächung der Röntgenstrahlung stärker als angenommen. Für die Vergleichbarkeit von DXA-Messungen muss immer derselbe Scan-Modus verwendet werden. Die Abbildungen 14 bis 16 veranschaulichen die Korrelation bei Knochenmineralgehalt, Knochenmineraldichte und R-Wert.

Tabelle 9

Beziehungen zwischen den Messergebnissen der Scan Modi „normal“ und „Pediatrie groß” R² (R-Square) und Standard Error of Estimation (SEE) für o.g. Parameter (Relations between results of measurements using Scan Modes "normal” and "Pediatric large" $\mathrm{R}^{2}$ (R-Square) and Standard Error of Estimation (SEE) and selected parameters))

\begin{tabular}{|c|c|c|c|c|c|c|c|}
\hline Genauigkeit & $\begin{array}{l}\text { Knochenmineral- } \\
\text { dichte }\end{array}$ & \multicolumn{2}{|c|}{$\begin{array}{l}\text { Knochenmineral- } \\
\text { gehalt }\end{array}$} & \multicolumn{2}{|c|}{$\begin{array}{l}\text { Knochenmineral- } \\
\text { anteil }\end{array}$} & \multirow{2}{*}{$\begin{array}{l}\begin{array}{l}\text { Mager- } \\
\text { weich- } \\
\text { gewebe }\end{array} \\
0.9870\end{array}$} & $\begin{array}{l}\text { Fett- } \\
\text { gewebe }\end{array}$ \\
\hline $\mathrm{R}^{2}$ & 0,9407 & 0,9661 & & 0,91 & & & 0,8181 \\
\hline SEE & 0,0159 & 56,8690 & & 0,09 & & 947,610 & 161,381 \\
\hline Genauigkeit & \multicolumn{2}{|c|}{ Magerweichgewebeanteil } & \multicolumn{2}{|c|}{ Fettgewebeanteil } & R-Wert & \multicolumn{2}{|c|}{ DXA-Gewicht } \\
\hline$\overline{\mathrm{R}^{2}}$ & \multicolumn{2}{|l|}{0,3906} & \multicolumn{2}{|c|}{0,4867} & 0,4744 & \multicolumn{2}{|l|}{0,9887} \\
\hline SEE & \multicolumn{2}{|l|}{0,3246} & \multicolumn{2}{|c|}{0,2020} & 0,0019 & \multicolumn{2}{|l|}{0,9459} \\
\hline
\end{tabular}




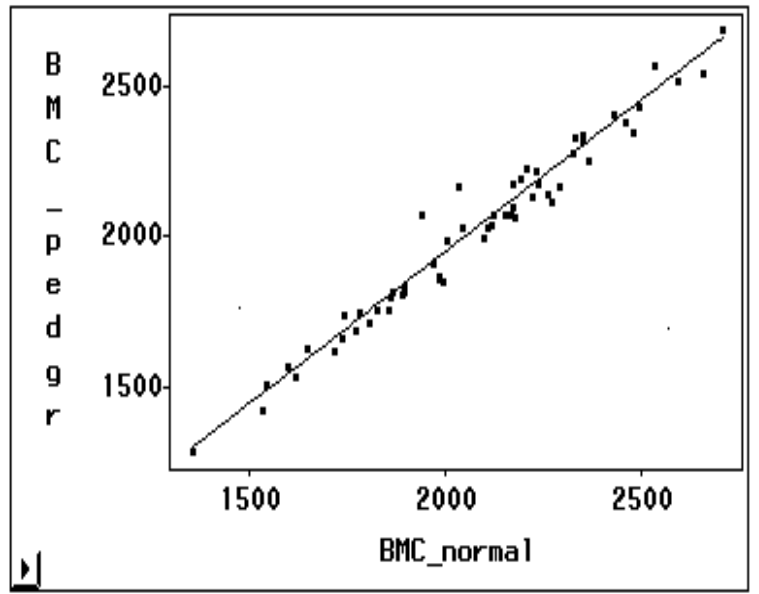

Abb. 14: Korrelation des Knochenmineralgehaltes von Abb. 15: Korrelation der Knochenmineraldichte von Scan Modus normal und Scan Modus Pediatrie groß Scan Modus normal und Scan Modus Pediatrie groß $(r=0,98)$ (Correlation of bone mineral content of scan $(r=0,97)$ (Correlation of bone mineral density of scan mode normal and scan mode pediatric large $(\mathrm{r}=0,98)) \quad$ mode normal and scan mode pediatric large $(\mathrm{r}=0,97))$

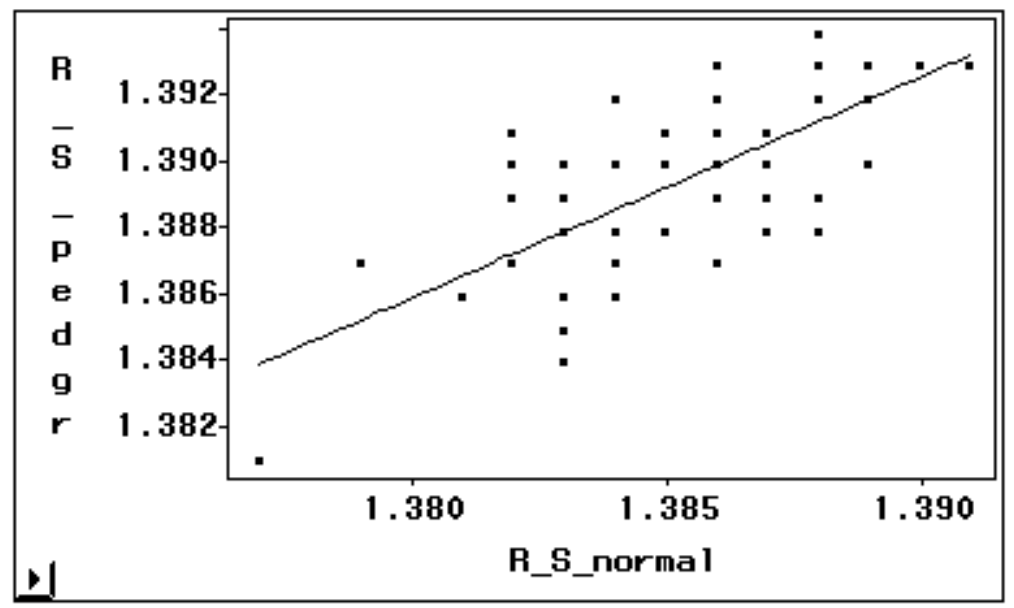

Abb. 16: Korrelation der R-Werte von Scan Modus normal und Scan Modus Pediatrie groß (r=0,69) (Correlation of R-Value of scan mode normal and scan mode pediatric large $(r=0,69))$

\section{Diskussion}

Die Unterschiede der einzelnen Genotypen der Kälber bezüglich ihrer Körperzusammensetzung sind signifikant. Erwartungsgemäß sind reinrassige DH-Kälber, was Knochenmineraldichte, Knochenmineralgehalt, Knochenmineralanteil, Fettgewebeanteil und das Gewicht betrifft, gegenüber den anderen Probanden im Nachteil. Kreuzungstiere erreichen dabei die höchsten Werte, wobei der DH-Anteil unterschiedlich hoch ist. Ein Heterosiseffekt ist hier anzunehmen, da Heterosis eine Überlegenheit der Kreuzungsnachkommen gegenüber ihren Reinzucht-Eltern darstellt. Heterosis kann für jede Eigenschaft wie folgt berechnet werden: Heterosis \% $=\left[\left(\right.\right.$ Durchschnitt der $\mathrm{F}_{1}-$ Kreuzung - Durchschnitt der Eltern) / Durchschnitt der Eltern] * 100 (KIELER, 2002). Die Heterosis für o.g. Messwerte liegt zwischen 3 \% und 5,89 \%. Bei der Knochenmineraldichte ist die Wahl des Vater- und Muttergenotyps entscheidend. In diesem Parameter erreichen Kälber mit DH-Vätern und FV-Müttern die höchsten Werte, aber nicht bei der reziproken Kreuzung, wobei aus der statistischen Analyse hervorgeht, dass der Muttergenotyp den größeren Effekt hat (F-Wert: 14,2 Muttergenotyp: p $\leq$ 0,05 vs. 5,8 Vatergenotyp: $\mathrm{p} \leq 0,05$ ). Beim Magerweichgewebsanteil erreichen reinrassige FV-Kälber den geringsten Wert, während reinrassige DH-Tiere alle anderen 
Genotypen übertreffen. ARRAYET et al. kamen 2002 bei ihrer Arbeit über das Wachstum von Holstein Kälbern bezüglich des Körpergewichts von DH-Kälbern in dieser Altersklasse (53,05 kg mit 30 Tagen) zu ähnlichen Ergebnissen wie in dieser Arbeit aufgeführt (50,48 kg mit 28 Tagen). Die Ergebnisse des Fettgewebsanteils dieser Arbeit stimmen nicht mit denen von BASCOM et al. (2002) überein, der Schlachtkörperhälften von Jersey-Bullenkälbern mit einem Hologic 4500A DXA Scanner untersucht hat und bei fünf Wochen alten Tieren für den Fettgewebsanteil Werte zwischen 12,79 \% und 20,85 \% erhielt. BUCKLEY et al. (1990) untersuchte die Körperzusammensetzung und Gewebeverteilung von Fleischjungrindern dreier Rassen (Hereford, Charolais, Simmental) im Alter von Geburt bis 14 Monate. Die Lebendgewichte der Simmentalkälber, die sehr gut mit Fleckviehkälbern vergleichbar sind, lagen in seiner Arbeit bei 38,5 kg für zwei Tage alte Kälber und bei 102,5 kg für drei Monate alte Tiere. Für zwei Tage alte Simmentalkälber bekam er nach chemischer Analyse Fettanteile von $2 \%$, bei drei Monate alten Tieren $7 \%$ Fettgewebeanteil. Dieses deckt sich mit den eigenen Ergebnissen und unterstreicht die Notwendigkeit der durchgeführten Korrektur der Werte anhand der R-Werte (s. Material und Methode 2.5.).

Großen Einfluss auf die Körperzusammensetzung haben die Genotypen der Elterntiere. Betrachtet man allein den Effekt der Vaterrasse bei den Parametern Knochenmineraldichte und -gehalt, sind Kälber von FV-Vätern den Kälbern von DH-Vätern in beiden Messergebnissen überlegen. Diese Genotypenunterschiede treffen auch bei den Muttertieren zu, wenn man isoliert die Knochenmineraldichte betrachtet. Bei Knochenmineralgehalt und Knochenmineralanteil erreichen die Kälber von Kreuzungsmüttern die höchsten Werte. Was den Magergewebsanteil betrifft, sind es Kälber von DH-Müttern, die den höchsten, und Kälber von FV-Müttern, die den niedrigsten Prozentanteil aufweisen.

Der tägliche Verbrauch von Kraftfutter im ersten Untersuchungsabschnitt unterliegt ebenso einem genetischen Einfluss der Muttertiere, wobei hier die Kälber von Kreuzungsmüttern am meisten Kraftfutter aufnehmen (98,4 \pm 11,0 g/Tag) und Kälber von FV-Müttern deutlich weniger fressen (73,1 \pm 7,7 g/Tag). Ähnliches liegt beim Vergleich der Kälbergenotypen für die tägliche Kraftfutteraufnahme vor. Reinrassige FVKälber nehmen im Vergleich zu den übrigen Genotypen am wenigsten Kraftfutter pro Tag auf (66,9 \pm 9,2 g vs. 87,3 \pm 11,9 g), was eventuell mit ihren im Vergleich zu den übrigen Tieren hohen Energiereserven in diesem Alter im Zusammenhang steht oder mit einer erhöhten Grundfutteraufnahme zu erklären wäre. Vergleichsliteratur zu dem Bereich der Kraftfutter- bzw. Heuaufnahme und daraus resultierende Zunahmen in der Altersklasse bis 50 Tage kann nicht angegeben werden, da Arbeiten mit so jungen Kälbern entweder ohne Heu und Kraftfutter (DIAZ et al. 2001, BLOME et al. 2003) durchgeführt wurden, oder beides ad libitum zur Verfügung stand (JENSEN et al. 2004). Allerdings muss beachtet werden, dass die Kraftfutteraufnahme/Tag in diesem Alter sehr niedrig war (durchschnittlich 85 g/Tag für alle Kälber).

Im zweiten Untersuchungsabschnitt hat die Laktationsnummer der Mutter einen deutlichen Einfluss auf die Aufnahme von Kraftfutter. Der Kraftfutterverbrauch liegt bei Kälbern aus Kühen der ersten beiden Laktationen hoch, ist bei Tieren aus Drittlaktierenden am niedrigsten und steigt dann für die Laktationsnummern 4 und 5 wieder leicht an. Dieses steht nicht in linerarem Zusammenhang mit den täglichen Gewichtszunahmen. Zwar haben Kälber von Erstlaktierenden die höchsten täglichen Zunahmen bezüglich Fettgewebe, Knochenmineralgehalt und DXA-Gewicht, doch es sind Kälber 
aus Kühen der vierten oder fünften Laktation, die die geringsten Zunahmen/Tag aufweisen.

Im ersten Untersuchungsabschnitt liegen die Unterschiede in den Zunahmen abhängig von der Laktationsnummer anders. Kälber aus Jungkühen zeigen die besten Knochenmineral-Zunahmen, aber sie bauen in der Tendenz Fettgewebe ab. Eine Erklärung für den tendenziellen Fettgewebsverlust ist schwierig zu geben, da bisher keine Arbeiten zu den Fettgewebszunahmen bei neugeborenen Kälbern existieren und sich damit keine Vergleichsmöglichkeit mit Ergebnissen aus anderen Arbeiten bieten. Möglich wäre auch eine unterschiedliche Hydratisierung des Weichgewebes bei den Probanden. PIETROBELLI et al. (1998) ermittelten in ihrer Studie, dass die unterschiedliche Hydratisierung von Weichgewebe bei DXA-Messungen zu Ungenauigkeiten bei der Fettgewebsbestimmung führt. Sie geben den Fehler bei der Fettgewebemessung durch DXA von $<1 \%$ bei Hydratationsänderungen von $1-5 \%$ an. Während des Wachstums ist die Hydratisierung des Gewebes mit dem Alter abnehmend, so dass damit eventuell die statistisch ermittelte Fettgewebsabnahme erklärbar wäre. Ob aber die Laktationsnummer, der Genotyp der Kälber oder der Genotyp der Elterntiere darauf einen Einfluss haben, kann nicht geklärt werden. Der Grund für die Knochenmineral-Zunahmen liegt möglicherweise in der abweichenden Kolostrumqualität von Kühen unterschiedlicher Laktationsnummern, die in diesem Alter hinsichtlich der Versorgung mit Ca und $\mathrm{P}$, die für das Knochenwachstum wichtig sind, eine bedeutende Rolle spielt. KUME et al. (1993) stellen in ihre Arbeit über den Effekt der Parität auf kolostrale Mineral-Konzentrationen bei Holstein-Kühen und den Wert von Kolostrum als Mineralstoffquelle für neugeborene Kälber fest, dass Kolostrum die Hauptquelle für Mineralstoffe für neugeborene Kälber nach der Geburt darstellt. Weiter fanden sie höhere Ca-Konzentrationen im Plasma bei Kälbern von Jungkühen als bei Kälbern von älteren Müttern. Tendenziell zeigen Kälber aus Kühen der dritten und fünften Laktation am ehesten eine Zunahme von Fettgewebe, wobei diese insgesamt verschwindend gering ist. Diese nehmen täglich das Meiste an Magerweichgewebe und Gesamtmasse zu. Ein weiterer interessanter Aspekt bei den täglichen Zunahmen liegt in dem Einfluss der Vaterrasse. Während zwischen den ersten beiden Scans Kälber von DH-Vätern die höheren Zunahmen erzielten, dreht sich dieses Verhältnis beim zweiten Untersuchungsabschnitt zugunsten der FV-Väter um. Während Tiere mit DH-Vätern bei Magerweichgewebeund DXWT-Zunahmen in beiden Zeiträumen relativ konstante Werte aufweisen, steigern die Kälber aus FV-Vätern ihre Zunahmen bei diesen Parametern um nahezu 100 g täglich im zweiten Untersuchungsabschnitt (s Tab. 6 und Tab. 8).

Betrachtet man die täglichen Zunahmen von Mehrlingen im Vergleich zu Einlingen, so wird offensichtlich, dass diese Tiere sofort anfangen, ihre geringeren Ausgangswerte mit höheren Tageszunahmen (421,8 g vs. 306,3 g) zu kompensieren. Dies gilt vor allem für den ersten Untersuchungsabschnitt. Dieses Ergebnis steht nicht im Einklang zu Ergebnissen von ECHTERNKAMP et al. (2002), der in seiner Arbeit Reproduktions-, Wachstums-, Mast- und Schlachtkörpereigenschaften von Zwillingen und Einlingen verschiedener Rassen vergleicht. In seiner Arbeit haben männliche Zwillingskälber (1,01 kg/Tag) weniger tägliche Zunahmen als Kälber aus Einlingsgeburten (1,08 kg/Tag) in einem Zeitraum von Geburt bis 172 Tage. Übereinstimmend sind die Ergebnisse zum Gewicht: Zwillingskälber wiegen von Geburt an weniger (36,8 kg Geburtsgewicht bei Echternkamp und 39,4 kg eigene Arbeit) als Kälber aus Einlingsgeburten (43,4 kg Geburtsgewicht bei ECHTERNKAMP und 49,78 kg eigene Arbeit). 
Insgesamt läßt sich die Dualenergie-Röntgenabsorptiometrie als ein geeignetes Verfahren beurteilen, die Körperzusammensetzung und das Wachstum von Kälbern zu untersuchen.

\section{Literatur}

ARRAYET, J.L.; OBERBAUER, A.M.; FAMULA, T.R.; GARNETT, I.; OLTJEN, J.W.; IMHOOF, J.; KEHRLI, M.E. jr.; GRAHAM, T.W.:

Growth of Holstein calves from birth to 90 days: The influence of dietary zinc and BLAD status. J. Anim. Sci., 80 (2002), 545-552

BASCOM, S.S.; HUFFARD, C.S.; NICKOLS- RICHARDSON, S.M.; HOVINGH, E.P.; JAMES, R.E.; MC GILLARD, M.L.: Analysis of body composition of Jersey bull calves fed varying levels of fat and protein with dual energy X-ray absorptiometry. J. Anim. Sci., 80 (2002), 336

BERTIN, E.; RUIZ, J.C.; MOUROT, J.; PEINIAU, P.; PORTHA, B.: Evaluation of dual-energy X-Ray absorptiometry for bodycomposition assessment in rats. J. Nutr., 128 (1998), 1550-1554

BLOME, R.M.; DRACKLEY, J.K.; MCKEITH, F.K.; HUTJENS, M.F.; MCCOY, G.C.: Growth, nutrient utilization, and body composition of dairy calves fed milk replacers containing different amounts of protein. J. Anim. Sci., 81 (2003), 1641-1655

BUCKLEY, B.A.; BAKER, J.F.; DICKERSON, G.E.; JENKINS, T.G.: Body composition and tissue distribution from birth to 14 months for three biological types of beef heifers. J. Anim. Sci., 68 (1990), 3109-3123

DIAZ, M.C.; VAN AMBURGH, M.E.; SMITH, J.M.; KELSEY, J.M.; HUTTEN, E.L.: Composition of Growth of Holstein Calves Fed Milk Replacer from Birth zo 105-Kilogramm Body Weight. J. Dairy Sci., 84 (2001), 830-842

ECHTENKAMP, S.E.; GREGORY, K.E.:

Reproductive, growth, feedlot, and carcass traits of twin vs single births in cattle. J. Anim. Sci., $\mathbf{8 0}$ (2002), E64-E73

ELLIS, K.:

Selected Body Composition Methods Can Be Used in Field Studies. J. Nutr., 131 (2001), 1589S-1595S

FINK, C.; COOPER, H.J.; HUEBNER, J.L.; GUILAK, F.; KRAUS, V.B.:

Precision and accuracy of a transportable dual-energy X-ray absorptiometry unit for bone mineral measurements in guinea pigs. Calcif Tissue Int., 70 (2002), 164-169

GALA PANIAGUA, J.; DIAZ-CURIEL, M.; DE LA PIEDRA GORDO, C.; CASTILLA RE, C.; TORRALBO

GARCIA, M.: Bone mass assessment in rats by dual-energy X-ray absorptiometry. Br. J. Radiol., 71 (1998), 754-758

HAMMAMI, M.; KOO, M.W.; KOO, W.W.; THOMAS, R.T.; RAKHMAN, D.:

Regional bone mass measurement from whole-body dual-energy X-ray absorptiometry scan. J. Clin. Densitom., 4 (2001), 131-136

JENSEN, M.B.:

Computer-Controlled Milk Feeding of Dairy Calves: Effects of Number of Calves per Feeder and

KIELER, K.C.: Number of Milk Portions on Use of Feeder and Social Behaviour. J. Dairy Sci., 87 (2004), 3428-3438

The Effects of Crossbreeding on Dairy Cattle Reproduction. Thesis of The Graduate Faculty, University of Wisconsin-Platteville, (2003)

KOO, W.W.; WALTERS, J.C.; HOCKMAN, E.M.:

Body Composition in Human Infants at Birth and Postnatally. J. Nutr., 130 (2000), 2188-2194

KOO, W.W.; HOCKMAN, E.M.; HAMMAMI, M.:

Dual energy X-ray absorptiometry measurements in small subjects: conditions affecting clinical measurements. J. Am. Coll. Nutr., 23 (2004), 212-219

KUME, S.; TANABE, S.;

Effect of Parity on Colostral Mineral Concentrations of Holstein Cows and Value of Colostrum as a Mineral Source for Newborn Calves. J. Dairy Sci., 76 (1993), 1654-1660

MITCHELL, A.D.; ROSEBROUGH, R.W.; CONWAY, J.M.:

Body composition analysis of chickens by dual energy x-ray absorptiometry. Poult. Sci., 76 (1997), 1746-1752

MITCHELL, A.D.; SCHOLZ, A.M.:

Dual-energy x-ray absorptiometry (DXA) analysis of growth and body composition of pigs of different ryanodine receptor genotypes. Arch. Tierz., Dummerstorf 40 (1997), 11-21

MITCHELL, A.D.; SCHOLZ, A.M.; CONWAY, J.M.: 
Body composition analysis of small pigs by dual-energy x-ray absorptiometry. J. Anim. Sci., 76 (1998), 2392-2398

MITCHELL, A.D.; SCHOLZ, A.M.; PURSEL, V.G.:

Total body and regional measurements of bone mineral content and bone mineral density in pigs by dual-energy x-ray absorptiometry. J. Anim. Sci., 79 (2001), 2594-2604

MITCHELL, A.D. ; SCHOLZ, A.M. ; PURSEL, V. :

Prediction of the in vivo Body composition of pigs based on crosssectional region analysis of dual energy X-ray absorptiometry (DXA) scans. Arch. Tierz., Dummerstorf 45 (2002), 535-545

NÜSKE, S. ; SCHOLZ, A.M. ; FÖRSTER, M. :

Studies on the Growth of the Claws in New Born Calves using Dual Energy X-Ray Absorptiometry (DEXA). Proceedings of the $12^{\text {th }}$ International Symposium on Lameness in Ruminants, Orlando, USA, (2002), 199-201

PICAUD, J.C.; NYAMUGABO, K.; BRAILlON, P.; LAPILLONNE, A.; ClARIS, O.; DELMAS, P.; MEUNIER, P.; SALLE, B.; RIGO, J.:

Dual-energy X-ray absorptiometry in small subjects: influence of dual-energy X-ray equipment on assessment of mineralization and body composition in newborn piglets. Pediatr. Res., 46 (1999), 772777

PIETROBELLI, A.; FORMICA, C.; WANG, Z.; HEYMSFIELD, S.B.:

Dual-energy $\mathrm{x}$-ray absorptiometry body composition model: review of physical concepts. American Physiological Society, (1996), E941-E951

PIETROBELLI, A.; WANG, Z.; FORMICA, C.; HEYMSFIELD, S.B.:

Dual-energy X-ray absorptiometrie: fat estimation errors due to variation in soft tissue hydration. American Physiological Society, (1998), E808-E816

POUILLES, J.M.; COLLARD, P.; TREMOLLIERES, F.; FRAYSSINET, P.; RAILHAC, J.J.; CAHUZAC, J.P.;

AUTEFAGE, A.; RIBOT, C.:

Accuracy and precision of in vivo bone mineral measurement in sheep using dual-energy X-ray absorptiometry. Calcif Tissue Int., 66 (2000), 70-73

SAS/STAT ${ }^{\circledR}$

User's Guide Volume 2, GLM-Varcomp, Version 8.2 TS Level 02M0, by SAS Institute Inc., Cary, NC, USA

SCHOLZ, A.M.:

In-vivo-Methoden zur Analyse von Muskelstoffwechsel und Körperzusammensetzung beim Schwein unter besonderer Berücksichtigung genetischer Einflüsse. Habil.-Schrift der Tierärztlichen Fakultät der Ludwig-Maximilians-Universität München, (2002), http://epub.ub.uni-muechen.de/archive/00000418/

SCHOLZ, A.M.; SOFFNER, P.; LITTMANN, E.; PESCHKE, W.:

Genauigkeit der Dualenergie-Röntgenabsorptiometrie (DXA) zur Ermittlung der Schlachtkörperzusammensetzung von Schweinehälften (kalt, 30-39 kg) anhand der EU-Referenzzerlegung. Züchtungskunde, 74 (2002), 376-391

SPEAKMAN, J.R.; BOOLES, D.; BUTTERWICK, R.:

Validation of dual-energy X-ray absorptiometry (DXA) by comparison with chemical analysis of dogs and cats. Int. J. Obes. Relat. Metab. Disord., 25 (2001), 439-447

SWENNEN, Q.; JANSSENS, G.P.; GEERS, R.; DECUYPERE, E.; BUYSE, J.:

Validation of dual-energy x-ray absorptiometry for determing in vivo body composition of chickens. Poult. Sci., 83 (2004), 1348-1357

TAMADA, A.; EMURA, S.; HAYAKAWA, D.; CHEN, H.; JAMALI, M.; YAMAHIRA YOSHIDATERASAWA,K.; ISONO, H.; SHOUMURA, S.:

Effect of immobilization on the ultrastructure of the golden hamster parathyroid gland. Okijamas Folia Anat. JPN., 74 (1998), 259-269

TURNER, A.S.; MALLINCKRODT, C.H.; ALVIS, M.R.; BRYANT, H.U.:

Dual-energy X-ray absorptiometry in sheep: experiences with in vivo and ex vivo studies. Bone, 17 (1995), 381-387

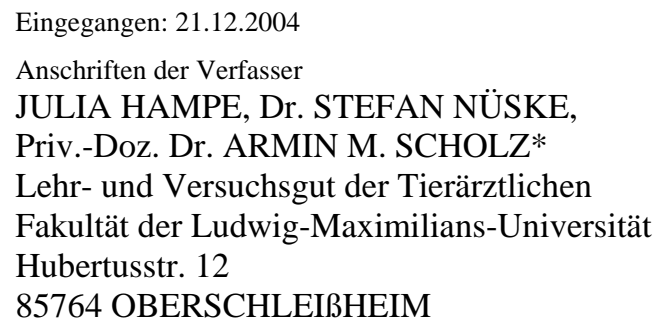

Akzeptiert: 20.06.2005

Univ.-Prof. Dr. Dr. habil. MARTIN FÖRSTER Institut für Tierzucht und Allgemeine Landwirtschaftslehre der Ludwig-Maximilians-Universität Veterinärstr.13 80805 MÜNCHEN 